\title{
An Improved Algebraic Method for Transit Signal Priority Scheme and Its Impact on Traffic Emission
}

\author{
Yanjie Ji, ${ }^{1}$ Bo Hu, ${ }^{1}$ Jing Han, ${ }^{1}$ and Dounan Tang ${ }^{2}$ \\ ${ }^{1}$ School of Transportation, Southeast University, Nanjing 210096, China \\ ${ }^{2}$ Department of Civil and Environmental Engineering, University of California-Berkeley, Berkeley, CA 94720-5800, USA \\ Correspondence should be addressed to Yanjie Ji; jiyanjie@seu.edu.cn
}

Received 4 March 2014; Accepted 14 April 2014; Published 11 May 2014

Academic Editor: Wuhong Wang

Copyright (c) 2014 Yanjie Ji et al. This is an open access article distributed under the Creative Commons Attribution License, which permits unrestricted use, distribution, and reproduction in any medium, provided the original work is properly cited.

\begin{abstract}
Transit signal priority has a positive effect on improving traffic congestion and reducing transit delay and also has an influence on traffic emission. In this paper, an optimal transit signal priority scheme based on an improved algebraic method was developed and its impact on vehicle emission was evaluated as well. The improved algebraic method was proposed on the basis of classical algebraic method and has improvements in three aspects. First, the calculation rules of split loss are more reasonable. Second, the delay caused by transit stations and queued vehicles can be considered. Third, measures for finding optimal ideal intersection interval are improved. By establishing a microscopic traffic emission simulation platform based on microscopic traffic simulation model VISSIM and the comprehensive modal emission model (CMEM), the traffic emissions can be evaluated. Then, an optimal transit signal priority scheme based on the traffic data collected in Changzhou city was developed and its impact on emission was simulated in the VISSIM-CMEM platform. Comparative analysis results showed that proposed scheme can outperform original scheme in the aspects of reducing emission and passenger delay and an average reduction of $25.0 \%$ on transit emission and relative decrease in overall traffic emission can be achieved.
\end{abstract}

\section{Introduction}

With the development of urban population, car ownership and usage increase every year, which has resulted in serious traffic congestion and acute environmental problem. As a major source of air pollution, vehicular traffic contributes considerably to the level of carbon monoxide (CO), nitrogen oxides $\left(\mathrm{NO}_{x}\right)$, and hydrocarbon $(\mathrm{HC})$ in many metropolitan areas [1]. Meanwhile, public transit priority policy has already become the core of urban traffic development strategy in China, which consists of a series of bus priority measures, such as transit lanes and transit signal priority. However, existing transit signal priority algorithms mainly focus on reducing traffic delay while paying little attention to evaluate its impact on traffic emissions. Some researches have pointed out that transit signal priority also offered a great potential benefit in reducing traffic emission [2].

Transit priorities' impact on traffic emission is actually a controversial topic. Alam and Hatzopoulou [3] argue that increasing transit ridership is not sufficient to achieve meaningful emission reductions at a regional level. Nonetheless, both of transit signal priority and signal coordination are efficient methods to reduce transit emission. Traffic signals at intersections impose significant impact on traffic emissions because they interrupt traffic flow and create additional deceleration, idle, and acceleration process [4]. Previous study developed plenty of methods to evaluate signal plan's impact on emission. Rakha et al. [5] showed that efficient signal coordination can reduce emissions up to $50 \%$ in a highly simplified scenario. Chen and $\mathrm{Yu}[6]$ integrated a comprehensive modal emission model (CMEM) [7] and VISSIM to evaluate vehicular emissions at signalized intersections and on bus lanes. Hirschmann and Fellendorf [8] developed a simulation toolbox to estimate pollutant emissions under different signal control strategies, and simulation results show 5-12\% emission reduction depending on pollutant types and signal control strategies. Tao et al. [9] also used field data to evaluate the effectiveness of signal coordination on 
reducing vehicle emissions during both peak and nonpeak hours. Lv and Zhang [10] investigate the effect of traffic signal coordination on emissions and compare it with their effects on operational performance measures of delay and stops.

Despite emission evaluating, previous studies have also attempted to develop signal timing optimization models to minimize both delays and emissions. Li et al. [11] optimized the cycle length and green splits for an isolated intersection to minimize a weighed sum of the delay, fuel consumption, and emission. Park et al. [12] developed signal timing optimization models to minimize the fuel consumption and vehicle emission based on microscopic simulation. Recently, Chen et al. [13] proved that the proposed model can not only keep intersection vehicle delay at a low level, but also effectively reduce vehicle emissions through instance validation. These models all focused on signal timing in isolated intersections. There are also a few studies trying to reduce emission in the whole network. Han et al. [14] consider an adaptive signal control problem on signalized network using Lighthill-Whitham-Richards (LWR) model with traffic-derived emission side constraints. Chou et al. [15] tied to improve transportation efficiency with latest green communication networking techniques in Intelligent Transportation Systems, by treating the Road Side Unit as a traffic signal control agent that can adaptively adjust traffic signal cycle in order to transport more passengers with increasing utilization of fuel consumption and reducing of pollutant emission of vehicles. However, these studies are mainly based on adaptive control methods, which are quite hard to be put into practice in nowadays China. For instance, in Changzhou, there are bus lanes and bus rapid transit (BRT). Although public transit trip rate is high in Changzhou, there is no sufficient condition to conduct adaptive signal control. This is a very common phenomenon in China.

Consequently we will focus on fixed transit signal coordination in this paper. There are mainly two methods to calculate optimal fixed signal offsets: to minimize vehicle delay or to maximize green wave bandwidth. Bandwidth is the amount of green time that can be used by a continuously moving platoon of vehicles through a series of intersections [16]. Graphical and algebraic methods are developed to find out a series of optimal offsets for every intersection that maximize the bandwidth. Classical algebraic method is able to work out an equitable signal timing solution for both two-way traffic, which also enjoys the advantages of easy understanding, easy implementation, and less computing time consumption. Classical algebraic method is especially appropriate for the signal timing for symmetric arteries where through movements make up the main traffic flow [17]. Therefore, this method is wildly applied in the design of realworld traffic control system and very popular in Chinese traffic engineering textbooks. However, it was found that there are several deficiencies in classical algebraic method. Li et al. [18] proposed that many problems existed in classical algebraic method such as the determination of value range of ideal intersection distance and the calculation of green wave bandwidth. Lu et al. [19] presented a new algebraic method of arterial road coordinate control for bidirectional green wave when traffic flow rates of two directions are not equal.
However, the defects of classical algebraic method itself have not been pointed out. $\mathrm{Hu}$ et al. [20] developed a modified graphic method and utilized it to find optimized signal timing plans and bus schedules according to the distances between adjacent intersections. Transit station was taken into consideration in that paper. But the difference of bandwidth between two directions resulted from the unsymmetrical distribution of transit stations near intersection was not been considered. According to previous studies, the three issues below need further study.

(i) More reasonable and practical algebraic method. Classical algebraic method has deficiencies in several aspects when used to calculate optimal signal coordination scheme. Previous studies have not clearly pointed all the deficiencies out or given effective solutions.

(ii) More realistic emission stimulation method for public transits. There are plenty of studies utilizing VISSIM and CMEM to evaluate traffic emission. However, few of them focus on public transits, where busload plays a pivotal role.

(iii) Transit signal priority that considering social vehicles. Transit signal priority in previous studies only focused on the public transit. The delay and emission of social vehicles were not considered. However, both public transit and social vehicles can benefit from the same fixed signal coordination plan at the same time.

Therefore this paper will focus on optimizing existing transit signal coordination algorithms and then evaluate their effects on traffic emissions. The remainder of this paper is organized as follows. In Section 2, the defects of classical algebraic method are analyzed and the improvement measures were proposed. A numerical example was demonstrated to show the difference between classical algebraic method and improved algebraic method. Afterwards a microscopic traffic emission simulation platform was established where the fluctuation of busload was taken into the calculation of vehicle emission. Section 3 is a case study based on traffic condition data collected in Changzhou, and a transit signal priority scheme is proposed in which social vehicles were also able to enjoy considerable bandwidth. Proposed scheme's impact on emission is then simulated and compared with real-world signal timing plan and signal timing method developed in the previous study. At last, conclusions and future research are included.

\section{Methodology}

2.1. Classical Algebraic Method. The core mission of classical algebraic method is to find out the optimal value of ideal intersection distance that leads to the maximal green wave bandwidth, after which signal timing scheme is determined based on the location of ideal intersections.

Supposing there are $N$ signalized intersections. Distances between every intersection and the first intersection are saved in vector $D=\left[D_{1}, D_{2}, \ldots, D_{N}\right]$. Splits of the intersections are 
saved in vector $G=\left[G_{1}, G_{2}, \ldots, G_{N}\right]$. The overall process of applying classical algebraic method is shown as below.

Step 1. Determine the common signal cycle length $C_{1}$ for all intersections.

Step 2. Determine the range of ideal intersection distance $a$ and iteration step size $s$ ( $s$ is generally equal to 10 meters):

$$
a \in\left[C_{1} \cdot \frac{v}{2}-M, C_{1} \cdot \frac{v}{2}+M\right],
$$

where, $v$ is vehicle's operating speed of through movements. $M$ is variation range of $a$.

Step 3. For every possible value of $a$, calculate the remainder vector $R^{\prime}=\left[R_{1}^{\prime}, R_{2}^{\prime}, \ldots, R_{N}^{\prime}\right]$, where $R_{i}^{\prime}$ is the remainder when $D_{i}$ is divided by $a$. Sort $R^{\prime}$ from the smallest to the largest into $R=\left[R_{1}, R_{2}, \ldots, R_{N}\right]$. Thereafter, calculate the judgment vector $J=\left[J_{1}, J_{2}, \ldots, J_{N-1}\right]$, where $J_{i}=R_{i+1}-R_{i}$. Save the maximal value of $J_{i}$ as $b$. Thus, there is a one-to-one correspondence between $a$ and $b$.

Step 4. Find out the maximal value of $b$ and its corresponding $a$ value $a^{*} \cdot a^{*}$ is just the optimal ideal intersection distance. Change the speed limit $v^{\prime}$ according to $a^{*}$ :

$$
v^{\prime}=\frac{2 a^{*}}{C_{1}}
$$

Step 5. Calculate signals offsets based on $a^{*}$ and intersections' relative position to ideal intersections.

As shown in Figure 1, there is a famous numerical example (NE1) that is referred to in many traffic control textbooks: there are 8 signalized intersections. $D=$ [0 m, $350 \mathrm{~m}, 750 \mathrm{~m}, 910 \mathrm{~m}, 1450 \mathrm{~m}, 1730 \mathrm{~m}, 2010 \mathrm{~m}, 2280 \mathrm{~m}$ ], $G=[55 \%, 60 \%, 65 \%, 65 \%, 60 \%, 65 \%, 70 \%, 50 \%], C_{1}=$ $80 \mathrm{~s}, v=40 \mathrm{~km} / \mathrm{h}, M=100 \mathrm{~m}, s=10 \mathrm{~m}$.

Therefore, $\left(C_{1} \cdot v\right) / 2=440 \mathrm{~m}$, and the possible values of $a$ are $340 \mathrm{~m}, 350 \mathrm{~m}, \ldots, 540 \mathrm{~m}$. After applying the classical algebraic method, the optimal ideal intersection distance $a^{*}$ is $500 \mathrm{~m}$. The new speed limit is $\left(2 a^{*}\right) / C_{1}=45 \mathrm{~km} / \mathrm{h}$. The green wave bandwidth is $30.5 \%$. The final signal timing results are shown in Table 1.

2.2. Improved Algebraic Method. Although enjoying the advantage of easy understanding, the offsets scheme found by classical algebraic method does not lead to the maximal green wave bandwidth. Taking the numerical example NE1 demonstrated above, the possible maximal bandwidth is actually around $36 \%$. Moreover, changing speed limit is impracticable in the urban area, for vehicles' operating speed is always stable in a certain road section. In addition, classical algebraic method built an ideal model that does not take delays into consideration, such as stop of buses and effect of queued vehicles at signals. Based on the deficiencies, an improved algebraic method is proposed, which is more practical in real-world traffic control system and can work out the actual maximal green wave bandwidth.

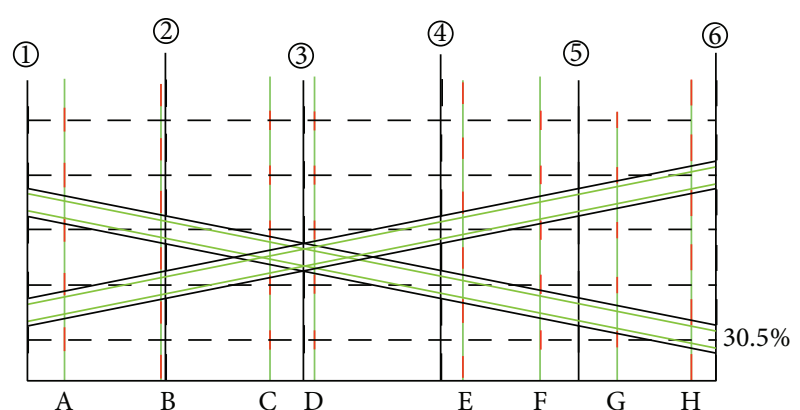

FIGURE 1: Numerical example NE1: time-space diagram of classical algebraic method.

2.2.1. The Calculation of Split Loss. The primary reason that classical algebraic method cannot solve out the maximal bandwidth is that its split loss calculation method is incorrect when the split is not equal to $50 \%$. In classical algebraic method, split loss is proportional to the distance between the real intersection and its nearest ideal intersection. As shown in Figure 2, ideal intersection interval $a$ is $500 \mathrm{~m}$, distance between intersection $A$ and its nearest ideal intersection (1), named as $d_{1}$, is $130 \mathrm{~m}$, and split is $50 \%$ for every signal. Green wave bandwidth among ideal intersections (1) and (2) is $50 \%$. Due to the existence of $d_{1}$, bandwidth is reduced by the length of $\mathrm{MN}$. For $\triangle O M N \sim \triangle O P Q, M N=P Q \cdot O N / O Q=$ $50 \% \times 130 / 500=13 \%$, which is the split loss of intersection $A$. The split loss is also called front-loss for this kind of split loss is in the front of original bandwidth. An example of backloss is also demonstrated in Figure 2. Therefore, split loss is also inversely proportional to ideal intersection interval. In algebraic method Step 4, we should find out the maximal value of $b / a$ instead of "find out the maximal value of $b$." However, calculation method of split loss shown above is correct only when split of every signal is equal to $50 \%$.

Given a more general situation, when split is lower or higher than $50 \%$, the split loss is determined by not only ideal intersection interval and the relative position of real intersection, but also the value of split. As shown in Figure 3, changing the splits of intersections $A$ and $B$, named $\lambda_{A}$ and $\lambda_{B}$, into $55 \%$ and $30 \%$, respectively. For vehicles moving to the right side of figure, direction- $\mathrm{R}$ for short, the intersection $A$ brings about a front-loss of $10.5 \%$ for it is ahead of its nearest ideal intersection, while intersection $B$ brings about a backloss of $16 \%$ since it is behind its nearest ideal intersection. Noticeably, as the split of $B$ is lower than $50 \%, B$ also causes a front-loss of $4 \%$ as well. We choose the largest front-loss and back-loss as the final split losses $L_{f}$ and $L_{b}$, in this case, $10.5 \%$ and $16 \%$, after which the green wave bandwidth GWB is calculated as GWB $=50 \%-10.5 \%-16 \%=23.5 \%$. It is the same case for vehicles moving to the left side of figure, where $A$ brings about a back-loss of $10.5 \%$ and $B$ brings about a front-loss of $16 \%$ and a back loss of $4 \%$. It also illustrates that algebraic method results in an equitable offset solution for both the two directions.

To sum up, the calculation rule of split loss in general situation is shown in Table 2. 
TABLE 1: Numerical example NE1: calculation result of classical algebraic method.

\begin{tabular}{|c|c|c|c|c|c|c|c|c|}
\hline Intersection number & $A$ & $B$ & C & $D$ & $E$ & $F$ & $G$ & $H$ \\
\hline Nearest ideal intersection number & (1) & (2) & (3) & (3) & (4) & (5) & (5) & (6) \\
\hline Relative location & Right & Left & Left & Right & Right & Left & Right & Left \\
\hline Split (\%) & 55 & 60 & 65 & 65 & 60 & 65 & 70 & 50 \\
\hline Split loss (\%) & 26 & 4 & 24 & 8 & 16 & 28 & 28 & 18 \\
\hline Effective Split (\%) & 29 & 56 & 41 & 57 & 44 & 37 & 42 & 32 \\
\hline Offset $(\%)$ & 72.5 & 20.0 & 67.5 & 67.5 & 20.0 & 67.5 & 65.0 & 25.0 \\
\hline
\end{tabular}

TABLE 2: Calculation rule of split loss.

\begin{tabular}{|c|c|c|c|}
\hline \multicolumn{2}{|c|}{ Ahead of nearest ideal intersection } & \multicolumn{2}{|c|}{ Behind of nearest ideal intersection } \\
\hline Front-loss $L_{f}$ & Back-loss $L_{b}$ & Front-loss $L_{f}$ & Back-loss $L_{b}$ \\
\hline $50 * d / a+(50-\lambda) / 2$ & $-50 * d / a+(50-\lambda) / 2$ & $-50 * d / a+(50-\lambda) / 2$ & $50 * d / a+(50-\lambda) / 2$ \\
\hline
\end{tabular}

After achieving the split loss of every signal on the artery, the maximal given wave bandwidth (GWB) of this artery can be solved out:

$$
\mathrm{GWB}=50 \%-L_{f}-L_{b}
$$

where $L_{f}=\max \left(L_{f I}\right)$ and $L_{f I}$ is the front-loss caused by intersection $I$. $L_{b}=\max \left(L_{b I}\right)$ and $L_{b I}$ is the back-loss caused by intersection $I$. Detailed numerical example NE1 of how to calculate split loss will be illustrated at the end of this chapter.

\subsubsection{Determining the Range of Ideal Intersection Distance and} Its Iteration Step Size. The final result of classical algebraic method is the optimal speed limit for the artery, where only vehicles traveling at this certain speed can enjoy the maximal green wave bandwidth. However, vehicles' operating speed is very difficult to control in reality. Therefore, we assume that all vehicles' operating speed is around their observed average speed and then change the value of common circle length according to optimal ideal intersection interval $a^{*}$. The final result of improved algebraic method is an optimal common circle length $C^{*}$ that leads to the maximal bandwidth when vehicles travel at their natural speed:

$$
C^{*}=\frac{2 a^{*}}{v},
$$

where $C^{*}$ is optimal common circle length, $a^{*}$ is optimal ideal intersection interval, and $v$ is vehicles' average operating speed.

Accordingly, the possible value of ideal intersection distance $a$ should also be changed. The range of $a$ should be

$$
a \in\left[C_{\min } \cdot \frac{v}{2}, C_{\max } \cdot \frac{v}{2}\right]
$$

where $C_{\min }=\max \left(C_{1 \min }, C_{2 \min }, \ldots, C_{N \min }\right) ; C_{\max }=$ $\min \left(C_{1 \max }, C_{2 \max }, \ldots, C_{N \max }\right) . C_{I \min }$ and $C_{I \max }$ are the lower and upper limit of intersection $I$ 's circle length. The iteration step size $s$ of ideal intersection distance $a$ should be $s=v / 2$. By this means, every iteration of $a$ value corresponds to the change in the common circle $C$ of one second.
2.2.3. Considering the Effect of Delay Caused by Queued Vehicles and Transit Station. Vehicles that enter the traffic stream between platoons will progress to the downstream signal. They form a queue that partially blocks the progress of the arriving platoon. These vehicles may include stragglers from the last platoon, vehicles that turned into the block from upstream intersections or vehicles that came out of parking lots or parking spots [16]. In addition, when designing signal coordination system for public transits, operating delay generated at transit station should also be taken into consideration. The ideal offset must be adjusted to allow for queued vehicles and public transits, so as to avoid unnecessary stops.

In the improved algebraic method, we take these situations into account by converting the delay caused by transit station and queued vehicles into additional distance between intersections:

$$
D_{\text {add } \_i}=D_{i}+\left(Q \cdot h+l_{1}+\mathrm{SD}\right) \cdot v,
$$

where $D_{\text {add } i}$ is the converted new distance between intersection $I$ and intersection $I+1 ; D_{i}$ is the original distance between intersection $I$ and intersection $I+1$; $Q$ is the average number of vehicles queued per lane on intersection $I+1 ; h$ is discharge headway of queued vehicles; $l_{1}$ is start-up loss time; SD is stop delay of public transit at transit stops on road section $I \sim I+1 ; v$ is average operating speed.

2.2.4. Finding Optimal Ideal Intersection Interval. Determining optimal ideal intersection interval $a^{*}$ is a critical step in classical algebraic method (Step 4), where $a^{*}$ is searched out from all possible values of $a$ by finding the largest corresponding $b$ value, which is the maximum of a's judgment vector $J$. As shown in several textbooks, the optimal ideal intersection interval $a^{*}$ of numerical example NE1 is $500 \mathrm{~m}$ and its corresponding $b$ value is 220 which leads to minimal split loss $280 / 500 / 2=28 \%$. In Step 3 of classical algebraic method, sorted remainder vector $R=[0,10,230,250,280,350,410,450]$; judgment vector $J=[10,220,20,30,70,60,40]$. Relative position of every intersection in an ideal intersection interval is plotted in Figure 4. However, it is maximal $b / a$ instead of $b$ that is 


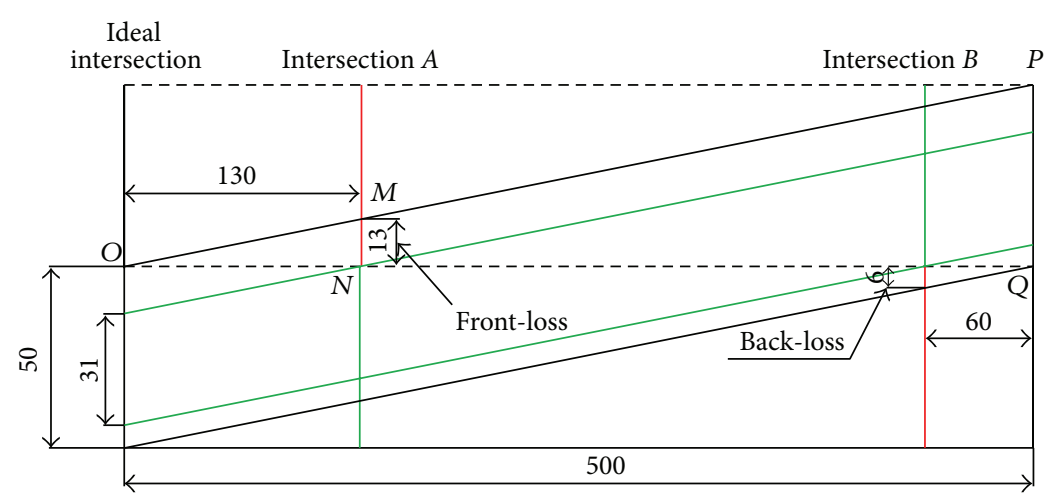

FIGURE 2: Example of split loss ( split $=50 \%)$.

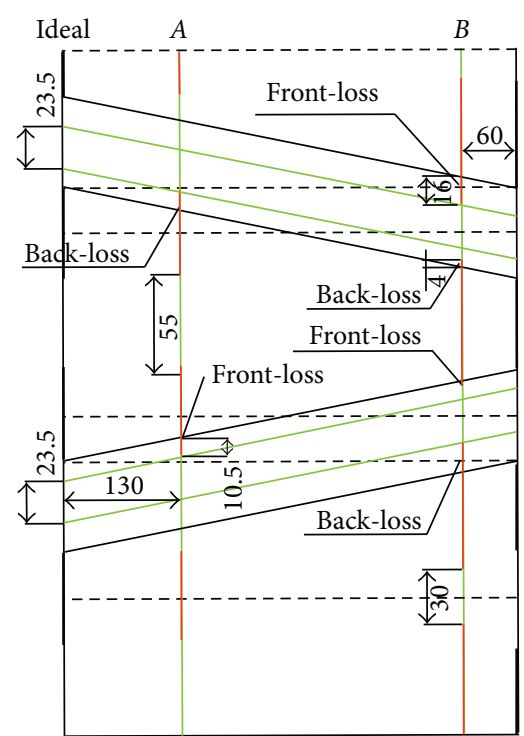

FIGURE 3: Example of split loss (split $\neq 50 \%$ ).

corresponding to minimal split loss. Also this method of finding optimal ideal intersection interval is correct only if the split of every intersection is $50 \%$, for only at that time split loss of a certain intersection is proportional to the distance between this intersection and its nearest ideal intersection.

In the improved algebraic method, intersections are arranged on a circle, whose circumference represents ideal intersection interval $a$. Relative distances between intersections in $a$ are indicated as several arc lengths, as shown in Figure 5(a). As split loss is also determined by green-timerate of each intersection, there is no simple or direct method to find out optimal ideal intersection interval $a^{*}$. Hence, we proposed an unsophisticated iteration process, which can be easily implemented within MATLAB programming, to calculate both front-loss and back-loss in a certain ideal intersection interval $a$.

(1) Plot relative position of $N$ intersects in ideal intersection interval as a circle. This circle is then rotated 180 degrees and overlapped on the original circle, as shown in Figure 5(b). The new circle is now divided into $2 N$ arcs, which means the iterative times equal $2 N$.

(2) Sequentially select one arc from these $2 N$ arcs and randomly choose one point in this arc as the position of ideal intersection. Draw a diameter from this point that cuts the circle into 2 semicircles and there should be both $N$ intersections on each semicircle. Intersections on one semicircle bring about front-loss and the other brings about backloss. Calculate split losses according to Table 2. N front-losses $\left[L_{f 1}, L_{f 2}, \ldots, L_{f N}\right]$ and $N$ back-losses $\left[L_{b 1}, L_{b 2}, \ldots, L_{b N}\right]$ will be achieved.

(3) Minimal split loss of ideal intersection interval $a$ equals $L_{a}=L_{f}+L_{b}$, where $L_{f}=\max \left(L_{f 1}\right.$, $\left.L_{f 2}, \ldots, L_{f N}\right), L_{b}=\max \left(L_{b 1}, L_{b 2}, \ldots, L_{b N}\right)$.

(4) Find out minimal $L_{a}$; its corresponding $a$ value is the optimal intersection interval $a^{*}$.

2.3. Numerical Example of Improved Algebraic Method. Using the same numerical example (NE1) mentioned in Section 2.1. Supposing minimal common circle length $C_{\text {min }}$ equals $60 \mathrm{~s}$, maximal common circle length $C_{\max }$ equals $100 \mathrm{~s}$, average operating speed equals $45 \mathrm{~km} / \mathrm{h}$, therefore the range of ideal intersection interval $a$ equals $\left[C_{\min } \cdot v / 2, C_{\max } \cdot v / 2\right]=$ [375 m, $625 \mathrm{~m}$ ]; iteration step size $s$ of ideal intersection distance $a$ equals $s=v / 2=6.25 \mathrm{~m}$. There are 40 possible $a$ values. The calculation result of several $a$ values' split loss is shown in Table 3.

For these $40 a$ values, $36.6 \%$ is the largest GWB value, which is also the actual maximal GWB value for this main stem. Therefore the optimal ideal intersection interval and common circle length of this numerical example are $481.25 \mathrm{~m}$ and $77 \mathrm{~s}$, respectively. The final signal timing results are shown in Figure 6. Thus, optimal offset of each signal can be calculated according to its relative position with its nearest ideal intersection. If its nearest ideal intersection's series number is an even number, the offset of this intersection equals $(50-\lambda / 2) \%$; if its nearest ideal intersection's series number is an odd number, the offset of this intersection equals $(100-\lambda / 2) \%$. 


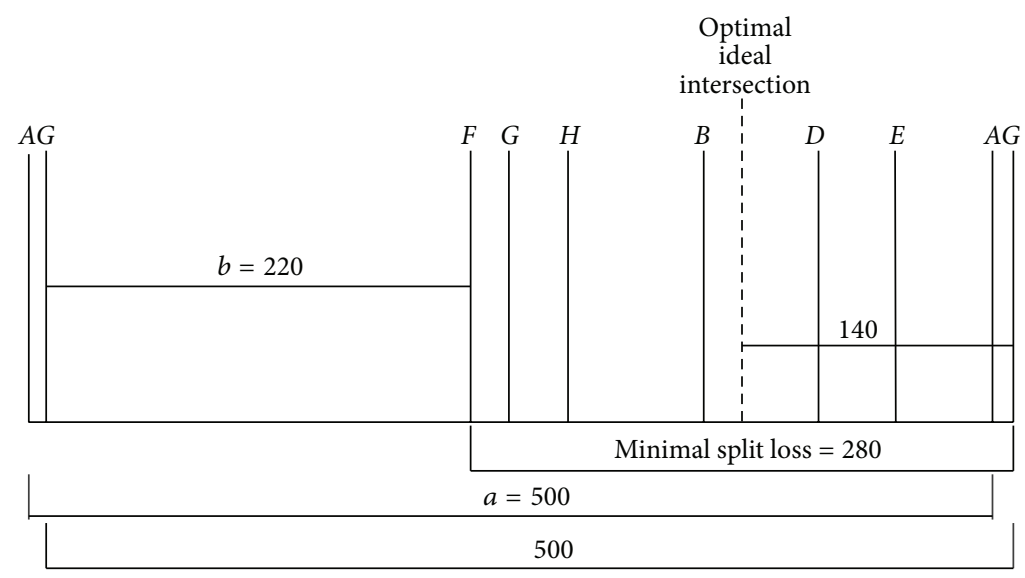

FIGURE 4: Finding optimal ideal intersection interval in classical algebraic method.

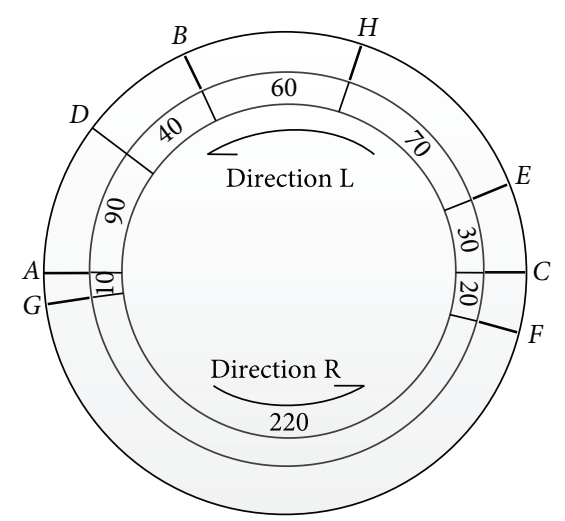

(a)

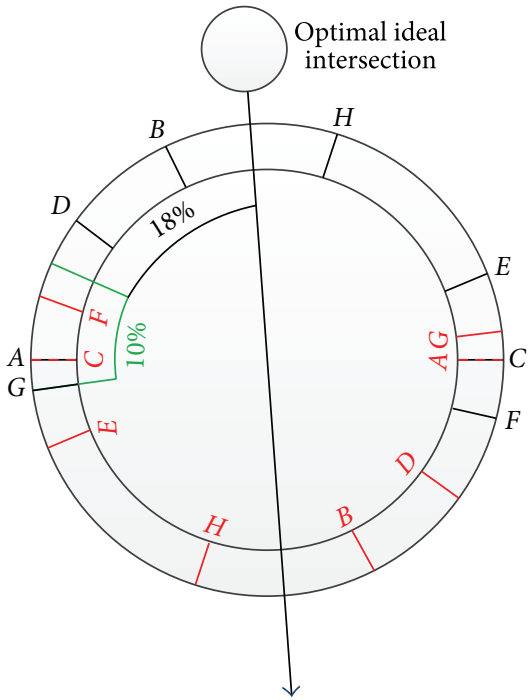

(b)

FIGURE 5: Finding optimal ideal intersection interval in improved algebraic method.

TABLE 3: Calculation of split loss in improved algebraic method.

\begin{tabular}{|c|c|c|c|c|c|c|c|c|c|c|c|c|}
\hline$a(\mathrm{~m})$ & & $C(\mathrm{~s})$ & $A$ & $B$ & $C$ & $D$ & $E$ & $F$ & $G$ & $H$ & Split loss (\%) & GWB (\%) \\
\hline \multirow{2}{*}{475} & \multirow{2}{*}{76} & $L_{f}(\%)$ & 4.6 & -11.1 & -21.4 & -4.6 & 4.7 & -18.3 & 8.7 & -2.9 & 8.7 & \multirow{2}{*}{34.9} \\
\hline & & $L_{b}(\%)$ & -9.6 & 1.1 & 6.4 & -10.4 & -14.7 & 3.3 & -28.7 & 2.9 & 6.4 & \\
\hline \multirow{2}{*}{481.25} & \multirow{2}{*}{77} & $L_{f}(\%)$ & 6.8 & -9.4 & -20.3 & -3.7 & 4.9 & -18.5 & 8.1 & -3.8 & 8.1 & \multirow{2}{*}{36.6} \\
\hline & & $L_{b}(\%)$ & -11.8 & -0.6 & 5.3 & -11.3 & -14.9 & 3.5 & -28.1 & 3.8 & 5.3 & \\
\hline \multirow{2}{*}{487.5} & \multirow{2}{*}{78} & $L_{f}(\%)$ & 12.6 & -4.0 & -15.4 & 1.0 & 8.8 & -14.9 & 11.3 & -1.0 & 12.6 & \multirow{2}{*}{36.3} \\
\hline & & $L_{b}(\%)$ & -17.6 & -6.0 & 0.4 & -16.0 & -18.8 & -0.1 & -31.3 & 1.0 & 1.0 & \\
\hline \multirow{2}{*}{493.75} & \multirow{2}{*}{79} & $L_{f}(\%)$ & -2.6 & -19.7 & -31.6 & -15.4 & -8.3 & -32.4 & -6.6 & -19.2 & -2.6 & \multirow{2}{*}{33.4} \\
\hline & & $L_{b}(\%)$ & -2.4 & 9.7 & 16.6 & 0.4 & -1.7 & 17.4 & -13.4 & 19.2 & 19.2 & \\
\hline \multirow{2}{*}{500} & \multirow{2}{*}{80} & $L_{f}(\%)$ & 20.5 & 3.0 & -9.5 & 6.5 & 13.0 & -11.5 & 14.0 & 1.0 & 20.5 & \multirow{2}{*}{30.5} \\
\hline & & $L_{b}(\%)$ & -25.5 & -13.0 & -5.5 & -21.5 & -23.0 & -3.5 & -34.0 & -1.0 & -1.0 & \\
\hline
\end{tabular}


TABLE 4: The mapping relationship of vehicle categories between VISSIM and CMEM.

\begin{tabular}{lccc}
\hline \multicolumn{2}{c}{ Vehicle categories } & $\begin{array}{c}\text { Vehicle types defined } \\
\text { in CMEM model }\end{array}$ & Technical characteristics \\
\hline \multirow{2}{*}{ Social vehicle } & Car & 5 & 3-way catalyst, FI (fuel-injected), >50 K miles, high power/weight \\
& LGV & 17 & Tier 1, light delivery truck, loaded vehicle weight: $3751-5750$ lbs \\
\hline \multirow{2}{*}{ Public transit } & Bus & 40 & Diesel-power, light delivery truck, gross vehicle weight: $>8500$ lbs \\
& BRT & 40 & Diesel-power, light delivery truck, gross vehicle weight: $>8500$ lbs \\
\hline
\end{tabular}

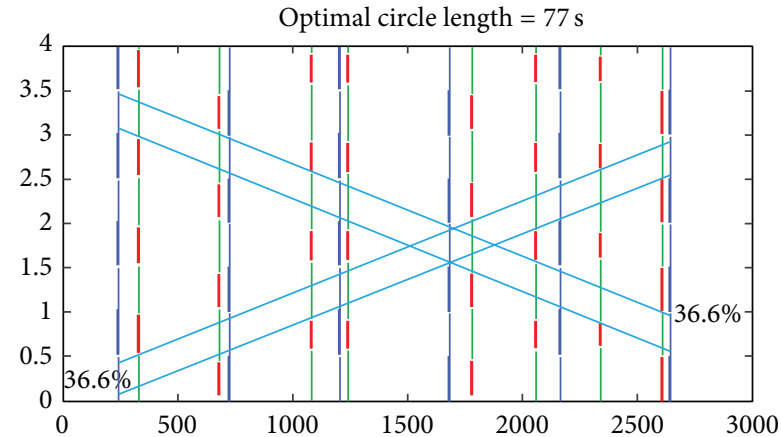

FiguRE 6: Numerical example NE1: time-space diagram of improved algebraic method.

\subsection{A Microscopic Traffic Emission Simulation Platform.} Many traffic simulation models have been used in combination with various statistical methods to quantify the traffic emissions. However, the results from such an approach are not very accurate nor can they capture the scenarios of dynamic traffic fluctuations [6]. Therefore, we adapted the microscopic simulation platform proposed by Chen and $\mathrm{Yu}$ [6], which can capture the dynamic traffic flows for estimating vehicle emissions, to evaluate the impact of the real world traffic on emissions. Based on their works, a more realistic communication interface is built up between the microscopic traffic simulation model VISSIM and the Comprehensive Modal Emission Model (CMEM), both of which have been proved to be effective simulation systems, to quantify the relationship between the traffic operations and emissions.

The key task in establishing the interface between VISSIM model and CEMEM model is to identify the mapping relationship of vehicle categories in these two models. Four types of vehicle will be studied in this paper: social vehicles consisting of car and Light Goods Vehicle (LGV) and public transits consisting of regular buses and Bus Rapid Transit (BRT) vehicles. These vehicles are defined in VISSIM and then three suitable vehicle categories are also selected from CMEM model based on the technical characteristics, as shown in Table 4. Noticeably, BRTs' working condition is assumed to be the same as regular buses while BRTs' net weight is considered twice as regular buses.' The fluctuation of load in public transit resulting from passengers going on and off is also taken into consideration, where weight of each person is regarded as $55 \mathrm{~kg}$.

\section{Case Study: BRT in Changzhou, China}

We utilize data collected in Changzhou to conduct this case study. Changzhou is located in the central zone of the Yangtze River delta in China, which was one of the very first cities in China to be equipped with Bus Rapid Transit (BRT). Road sections selected in this case study are a part of a main BRT line in Changzhou, from Hehai Road to Feilong Road, whose length is $3.8 \mathrm{~km}$. This main stem goes across both the urban fringe and the downtown, where data collected can typically represent the traffic condition of the whole city.

From 19th November, 2011, to 28th December, 2011, research groups of our school researched the city for three times and collected the statistics for the subsequent study. Statistics we had to collect in our research could be approximately categorized into three types. Each of them is the essential data of the intersection, the operating data of BRT, and also the fundamental data along the section. On 19th November, the advance research was carried out, which did all the preparation for the next research. Next, the essential data of the intersection, including traffic flow, original signal timing data, and the fundamental data along the section, including road length and intersection channelization, were measured artificially from 27th December to 28th December. Finally, from 27th December to 28th December, data about the operation of BRT, including service time at each transit station and departure frequency, are measured at both the peak hour and the no-peak time.

In this case study, aiming to reduce traffic delay and emissions at the same time, we proposed a method to design optimal offset schemes that maximize the green wave bandwidths for both general vehicle and public transits based on the improved algebraic method. Afterwards, this offset scheme we proposed was compared with real-world offset scheme and another transit signal priority scheme in the aspect of average passenger delay and emission.

3.1. Design Signal Coordination Scheme. Based on the collected data, there are 8 intersections in this case study, distances between every intersections and the first intersection are saved in vector $D=[0 \mathrm{~m}, 782 \mathrm{~m}, 1110 \mathrm{~m}, 1674 \mathrm{~m}$, $2383 \mathrm{~m}, 2747 \mathrm{~m}, 3513 \mathrm{~m}, 3763 \mathrm{~m}$ ] (from north to south). Signal timing scheme of each intersection is calculated based on collected traffic flow and the signal timing method proposed in [21], which is able to achieve a balance between minimizing average passenger delay and minimizing total vehicle emissions. Thereafter, key parameters of 


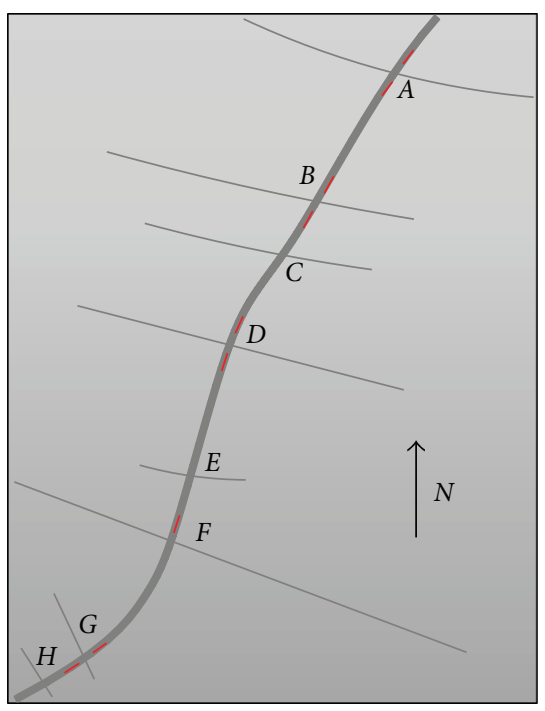

Figure 7: Road network in VISSIM.

each intersection can be achieved, where splits are saved in vector $G=[38.2 \%, 37.5 \%, 43.3 \%, 35.5 \%, 54.0 \%, 45.0 \%$, $62.8 \%, 65.8 \%$ ] (from north to south); common circle length $C_{\min }$ equals $113 \mathrm{~s}$; maximal common circle length $C_{\max }$ equals $167 \mathrm{~s}$; average operating speed of general vehicle equals $45 \mathrm{~km} / \mathrm{h}$; average operating speed of BRT equals $50 \mathrm{~km} / \mathrm{h}$ (there are transit lanes in the center of main stem; detailed road cross-sectional figure can be viewed in [22]).

Road network is built in VISSIM as shown in Figure 7 after simplification, where deep red lines represent transit stops. It can be observed that in several road sections the arrangement of transit stop is unsymmetrical. For instance near intersection $B$, transit stop severing for buses traveling to north is set in the north of intersection $B$ while transit stop severing for buses traveling to south is set in south of intersection $B$. According to (6), equivalent distance of BA $D_{\text {add } A B}$ is not equal to the distance of $A B$, which means there exists different bandwidths in the same offset scheme for public transits traveling to north and south. Thus there are actually three different bandwidths under the same offset scheme, $G_{\text {car }}$ (GWB of general vehicles), $G_{\text {bus_s }}$ (GWB of public transit traveling to south), and $G_{\text {bus } n}$ (GWB of public transit traveling to north). Dwell time including a constant part and a random part. The constant part represents the time consumption when the passenger side door opened and closed. The random part represents the time consumption when passengers go on and off. It is supposed to follow the normal distribution, whose mean and variance are extracted from collected data. For example, the dwell time of BRT line 1 heading to north in the station near intersection $B$ is $T c+T x$, where $T c=5 \mathrm{~s} ; T x \sim N(21 \mathrm{~s}, 16 \mathrm{~s})$. We calculated all the possible results of these three green wave bandwidths out under different common circle lengths and signal coordination schemes, which are shown in Figure 8. Possible solutions under the same common circle length and signal coordination scheme are colored in the same name. We consider the priority of public transit by allocating

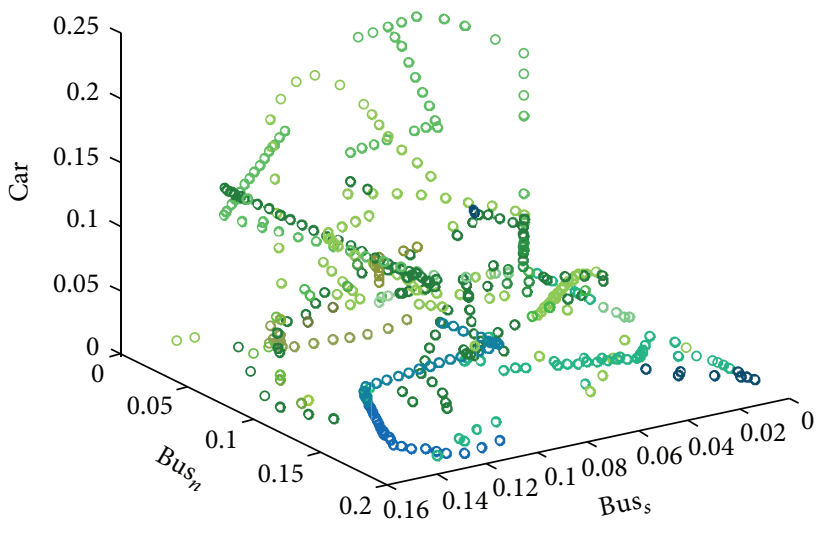

FIGURE 8: All possible solutions of bandwidth.

reasonable weights on GWB of general vehicles and public transit:

$$
W=Q_{\text {bus }} * \frac{P_{\text {bus }}}{\left(Q_{\text {car }} * P_{\text {car }}\right)},
$$

where $W$ is the weight factor of public transit; $Q_{\text {bus }}$ and $Q_{\text {car }}$ are the average flow rate of public transit and general vehicle on all intersections; $P_{\text {bus }}$ and $P_{\text {car }}$ are the average passengers per vehicle of public transit and general vehicle on all intersections.

The target GWE equals $G_{\text {car }}+W\left(G_{\text {bus_s }}+G_{\text {bus_n } n}\right)$. In peak hour $W p=1.09$ while in no-peak hour $W n=1.05$. As these two values are very closed, they actually lead to the same optimal signal coordination scheme as shown in Table 4. The optimal common circle length is $152 \mathrm{~s}$, where $G_{\text {car }}=11.1 \%, G_{\text {bus } s}=12.2 \%$, and $G_{\text {bus } n}=15.4 \%$. To assess this proposed signal coordination scheme's impact on average passenger delay and emission, the other two schemes are also shown in Table 5: the real-world signal timing scheme and another transit signal priority scheme [20], which only maximizes the bandwidth for public transit and does not consider the differences between bandwidths for buses heading to different directions.

3.2. Evaluating Vehicle Emissions. In this study, a comparison between three signal timing schemes is used to evaluate their impacts on traffic emissions. Setting signal timing plan for every intersection in VISSIM following Table 4, simulation results of every vehicle' velocity and acceleration in every simulation second can be achieved. Traffic flow data collected from 4:30 pm to 5:30 pm in Changzhou is utilized and simulation time length is $3600 \mathrm{~s}$. Thereafter, CMEM is utilized to calculate traffic emission and fuel consumption based on these simulation results. Table 6 illustrates the simulation results of different emission species and fuel consumption for different signal coordination schemes. Figure 9 shows the change of emission after conducting Scheme 1 and Scheme 3 compared to real-world signal coordination scheme (Scheme 2).

As shown in Table 6 and Figure 9, after conducting proposed scheme calculated by improved algebraic method (Scheme 1), the emissions generated by public transit, including regular buses and BRTs, are averagely reduced by $25.0 \%$, 
TABLE 5: Case study: transit signal priority scheme based on improved algebraic method.

\begin{tabular}{|c|c|c|c|c|c|c|c|c|c|}
\hline \multirow{2}{*}{\multicolumn{2}{|c|}{$\begin{array}{c}\text { Intersection number } \\
\text { Split (\%) }\end{array}$}} & $A$ & $B$ & $C$ & $D$ & $E$ & $F$ & $G$ & $H$ \\
\hline & & 38 & 38 & 43 & 34 & 54 & 45 & 63 & 66 \\
\hline \multirow{2}{*}{$\begin{array}{l}\text { Scheme 1-our transit } \\
\text { signal priority scheme }\end{array}$} & Offset (\%) & 48 & 125 & 120 & 57 & 36 & 119 & 105 & 27 \\
\hline & Circle length (s) & 152 & 152 & 152 & 152 & 152 & 152 & 152 & 152 \\
\hline \multirow{2}{*}{$\begin{array}{l}\text { Scheme 2-real world } \\
\text { scheme }\end{array}$} & Offset (\%) & 42 & 106 & 54 & 19 & 22 & 4 & 108 & 31 \\
\hline & Circle length (s) & 120 & 160 & 130 & 130 & 146 & 73 & 146 & 146 \\
\hline \multirow{2}{*}{$\begin{array}{l}\text { Scheme 3-Hu's transit } \\
\text { signal priority scheme }\end{array}$} & Offset (\%) & 33 & 86 & 30 & 88 & 24 & 82 & 20 & 71 \\
\hline & Circle length (s) & 106 & 106 & 106 & 106 & 106 & 106 & 106 & 106 \\
\hline
\end{tabular}

TABLE 6: Simulation results of emission, fuel consumption, and passenger delay.

\begin{tabular}{lccccccc}
\hline Scheme & Vehicle type & $\mathrm{HC}(\mathrm{g} / \mathrm{km})$ & $\mathrm{CO}(\mathrm{g} / \mathrm{km})$ & $\mathrm{NO}_{x}(\mathrm{~g} / \mathrm{km})$ & $\mathrm{Fuel}(\mathrm{g} / \mathrm{km})$ & $\mathrm{CO}(\mathrm{g} / \mathrm{km})$ & $\mathrm{Delay}(\mathrm{s} / \mathrm{p})$ \\
\hline \multirow{2}{*}{ Scheme 1 } & Social vehicles & 0.980 & 4.50 & 0.88 & 150.3 & 570.51 & 17.47 \\
& Public transit & 1.351 & 51.7 & 13.48 & 392.6 & 1987.79 & 2.32 \\
\hline \multirow{2}{*}{ Scheme 2 } & Social vehicles & 0.962 & 4.34 & 0.84 & 145.6 & 555.01 & 13.37 \\
& Public transit & 1.84 & 68.37 & 18.12 & 518.8 & 2627.78 & 2.77 \\
\hline \multirow{2}{*}{ Scheme 3 } & Social vehicles & 0.995 & 4.69 & 0.97 & 156 & 603.75 & 21.95 \\
& Public transit & 1.457 & 53.96 & 13.74 & 398.3 & 2032.11 \\
\hline
\end{tabular}

while the emission of social vehicles slightly increased. It is because public transits' emissions are strongly dependent on vehicles' operating modes, especially during acceleration, as shown in Figure 10. Expanding green wave bandwidth for public transit can reduce the acceleration process which therefore results in less emission. The level of service of public transit is also improved for passenger delay decreased by $16 \%$. Compared to Hu's method (Scheme 3 ), emission species generated by public transit are lower in scheme 1 . Meanwhile, social vehicles emission observably decreased and the social vehicles delay also become lower. In general, through these one-hour simulations, the implementation of Scheme 1 reduces HC emission by $993.5 \mathrm{~g}$, CO by $6146.0 \mathrm{~g}$, $\mathrm{NO}_{x}$ by $391.2 \mathrm{~g}$, fuel consumption by $32506.3 \mathrm{~g}$, and $\mathrm{CO}_{2}$ by $63476.4 \mathrm{~g}$ compared to real-world signal timing scheme (Scheme 2). Compared to signal coordination scheme that only maximal green wave bandwidth for public transit, applying our scheme (Scheme 1), can reduce HC emission by $260.1 \mathrm{~g}, \mathrm{CO}$ by $1358.0 \mathrm{~g}, \mathrm{NO}_{x}$ by $749.9 \mathrm{~g}$, and fuel consumption by $6522.3 \mathrm{~g}$ while the emission of $\mathrm{CO}_{2}$ increased by $14136.0 \mathrm{~g}$.

\section{Conclusion}

This paper developed an improved algebraic method to calculate optimal transit signal priority scheme. By establishing a microscopic traffic emission simulation platform, proposed transit signal priority scheme's effects on emission were evaluated and compared in the aspect of emission, fuel consumption, and passenger delay. Based on the realworld traffic condition data collected in Changzhou, a case study was also presented to evaluate the performance of the proposed method. The main contributions of this paper are listed below.

(i) Defects of classical algebraic method were pointed out and an improved algebraic method was proposed.
Relative improvements include accurate calculating split loss in general situation, considering the effects of delays caused by queued vehicles and transit station, and measures of finding optimal ideal intersection interval. Numerical example shows that the proposed improved algebraic method is more precise and practical.

(ii) A microscopic traffic emission simulation platform based on microscopic traffic simulation model VISSIM and the comprehensive modal emission model (CMEM) was established to evaluate traffic emissions. Fluctuation of public transit load weight is also taken into account, which results in more realistic public transit emission simulation.

(iii) Based on the survey conducted in Changzhou, an optimal transit signal priority scheme was proposed. The scheme takes both social vehicle and public transit into the calculation of optimal offset scheme and maximal green wave bandwidth. The different characteristics of vehicles traveling in different directions are also considered.

(iv) Proposed transit signal priority scheme's impact on emission was assessed. Based on the traffic emission simulation platform, emissions and fuel consumptions are simulated. Results show that the proposed scheme precedes previous studies in the aspect of overall emission and passenger delay. Compared to the real-world signal timing plan in Changzhou, the proposed scheme can reduce $\mathrm{HC}$ emission by $993.5 \mathrm{~g}$, CO by $6146.0 \mathrm{~g}, \mathrm{NO}_{x}$ by $391.2 \mathrm{~g}$, fuel consumption by $32506.3 \mathrm{~g}$, and $\mathrm{CO}_{2}$ by $63476.4 \mathrm{~g}$.

However, because of the limitations of the survey data and the algorithm, the research has several aspects to be improved in the future. Level of service of the proposed optimal transit 


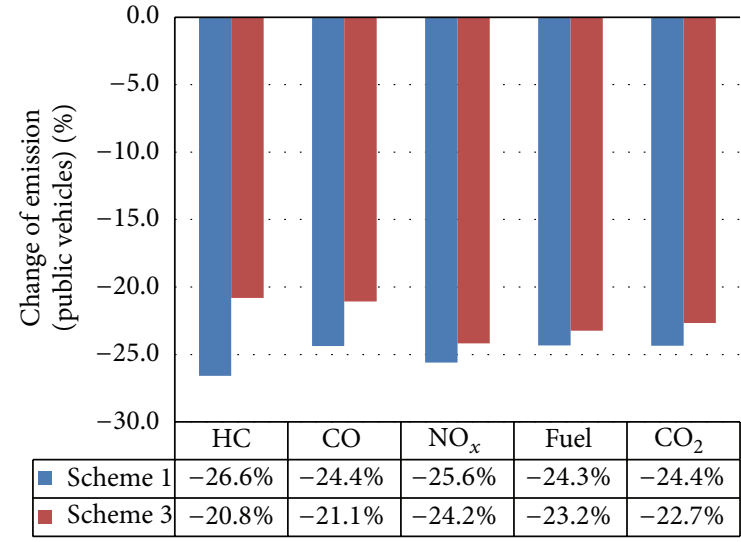

(a)

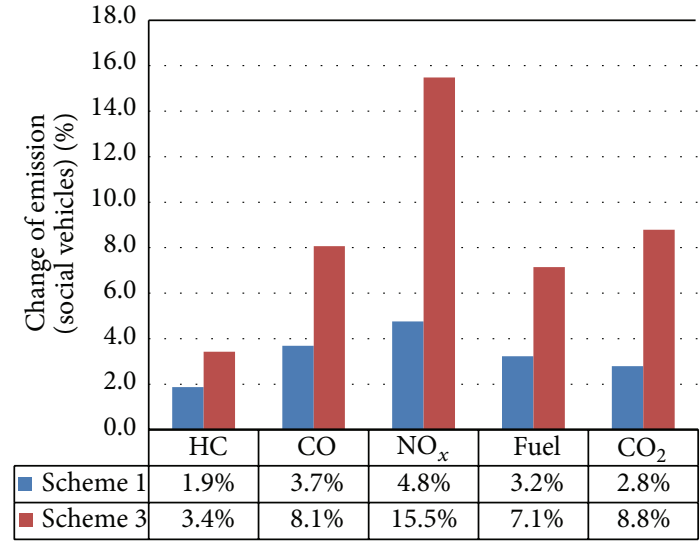

(b)

FIGURE 9: (a) Change of emission of public transit compared to real-world signal plan. (b) Change of emission of social vehicle compared to real-world signal plan.

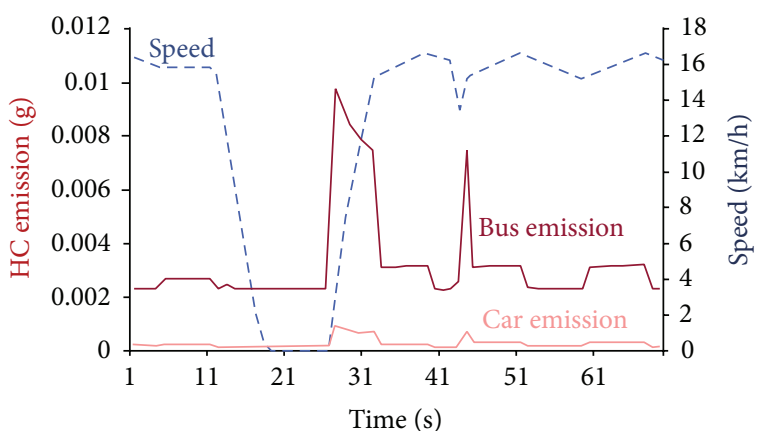

FIGURE 10: HC emission of public transit and social vehicle.

signal priority scheme can be further improved by changing some intersections' circle length or split and a succeeding signal timing approach can be developed. Using vehicle type 40 in CMEM to represent public transit is not precisely enough, which can be improved by conducting experiments to collect realistic emission data.

\section{Conflict of Interests}

The authors declare that there is no conflict of interests regarding the publication of this paper.

\section{Acknowledgments}

The key project of national natural science foundation of China (no. 51338003) and the financial support from the National Key Basic Research Programme of China (no. 2012CB725402) are gratefully acknowledged.

\section{References}

[1] A. R. Sharma, S. K. Kharol, and K. V. S. Badarinath, "Influence of vehicular traffic on urban air quality-a case study of
Hyderabad, India," Transportation Research D: Transport and Environment, vol. 15, no. 3, pp. 154-159, 2010.

[2] Z. Wang, J. Wang, and D. He, "Transit policies and potential carbon dioxide emission impacts," Transportation Research Record, vol. 2287, no. 1, pp. 98-104, 2012.

[3] A. Alam and M. Hatzopoulou, "A microscopic simulation of transit bus emissions under transit signal priority and alternative technology," in Proceedings of the 92nd Annual Meeting of the Transportation Research Board, 2013.

[4] A. Y. Bigazzi and M. A. Figliozzi, "The role of heavy-duty freight vehicles in reducing emissions on congested freeways with elastic travel demand functions," in Proceedings of the 92nd Annual Meeting of the Transportation Research Board, 2013.

[5] H. Rakha, M. van Aerde, K. Ahn, and A. A. Trani, "Requirements for evaluating traffic signal control impacts on energy and emissions based on instantaneous speed and acceleration measurements," Transportation Research Record, no. 1738, pp. 56-67, 2000.

[6] K. Chen and L. Yu, "Microscopic traffic-emission simulation and case study for evaluation of traffic control strategies," Journal of Transportation Systems Engineering and Information Technology, vol. 7, no. 1, pp. 93-99, 2007.

[7] M. Barth, F. An, T. Younglove et al., Comprehensive Modal Emission Model (CMEM), Version 2.0 User's Guide, University of California, Riverside, Calif, USA, 2000.

[8] K. Hirschmann and M. Fellendorf, "A toolbox to quantify emission reductions due to signal control," in Proceedings of the 89th Annual Meeting of the Transportation Research Board, 2010.

[9] F. Tao, Q. Shi, and L. Yu, "Evaluation of effectiveness of coordinated signal control in reducing vehicle emissions during peak hours versus nonpeak hours," Transportation Research Record, vol. 2233, no. 1, pp. 45-52, 2011.

[10] J. Lv and Y. Zhang, "Effect of signal coordination on traffic emission," Transportation Research D: Transport and Environment, vol. 17, no. 2, pp. 149-153, 2012.

[11] X. Li, G. Li, S.-S. Pang, X. Yang, and J. Tian, "Signal timing of intersections using integrated optimization of traffic quality, emissions and fuel consumption: a note," Transportation Research D: Transport and Environment, vol. 9, no. 5, pp. 401407, 2004. 
[12] B. B. Park, I. Yun, and K. Ahn, "Stochastic optimization for sustainable traffic signal control," International Journal of Sustainable Transportation, vol. 3, no. 4, pp. 263-284, 2009.

[13] H. Chen, R. Bai, J. Ma, and D. Wang, "Research on intersection signal timing model considering emissions effects," in CICTP, 2012: Multimodal Transportation Systems-Convenient, Safe, Cost-Effective, Efficient, ASCE, 2012.

[14] K. Han, T. L. Friesz, H. Liu, and T. Yao, "A robust optimization approach for dynamic traffic signal control with emission constraints," http://arxiv.org/abs/1211.4865.

[15] L. D. Chou, B. T. Deng, and K.-W. Kuo, "A passenger-based adaptive traffic signal control mechanism in Intelligent Transportation Systems," in Proceedings of the 12th International Conference on ITS Telecommunications (ITST '12), IEEE, 2012.

[16] W. R. McShane and R. P. Roess, Traffic Engineering, Prentice Hall, 1990.

[17] J. Chen, L. Xu, S. Zhu, G. Zhang, H. Wang, and S. Li, Traffic Control and Management, China Communication Press, 2012.

[18] L. Li, J. M. Xu, and K. Lu, "An improved algebraic method of green wave based on one-phase-one-approach," Journal of Transport Information and Safety, vol. 27, no. 3, pp. 64-67, 2009.

[19] K. Lu, J.-M. Xu, and Y.-S. Li, "Algebraic method of arterial road coordinate control for bidirectional green wave under signal design mode of one-phase-one-approach," China Journal of Highway and Transport, vol. 23, no. 3, pp. 95-101, 2010.

[20] X. Hu, W. Wang, and S. Chen, "Optimization control method of traffic signal timings and bus schedule for one-route one-line and run-through bus system," Journal of Southeast University (Natural Science Edition), vol. 41, no. 4, pp. 145-150, 2011.

[21] L. Zhang, Y. Yin, and S. Chen, "Robust signal timing optimization with environmental concerns," Transportation Research C: Emerging Technologies, vol. 29, pp. 55-71, 2013.

[22] Y. C. Lu and M. Yu, "Comparison of BRT systems in four Chinese cities: Beijing, Changzhou, Xiamen and Jinan," in Sustainable Transportation Systems: Plan, Design, Build, Manage, and Maintain, ASCE, 2012. 


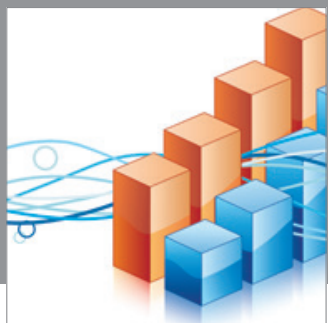

Advances in

Operations Research

mansans

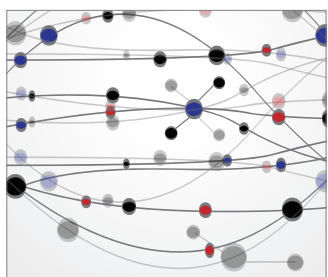

The Scientific World Journal
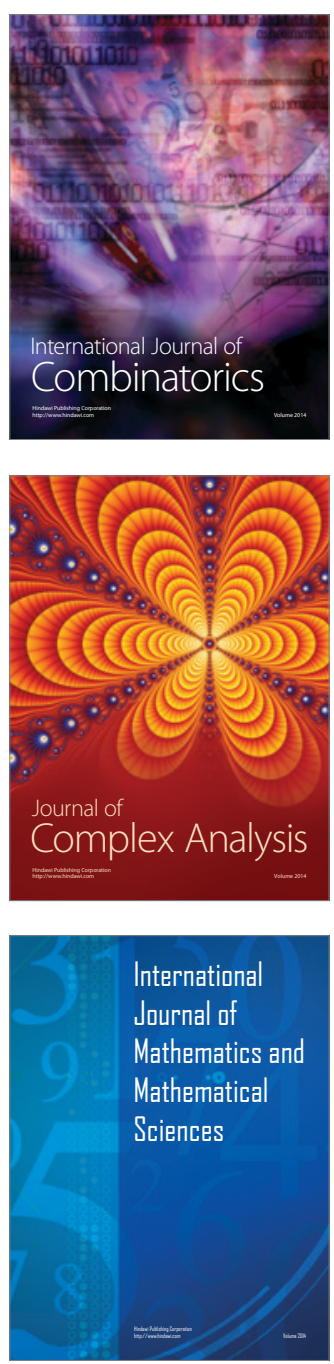
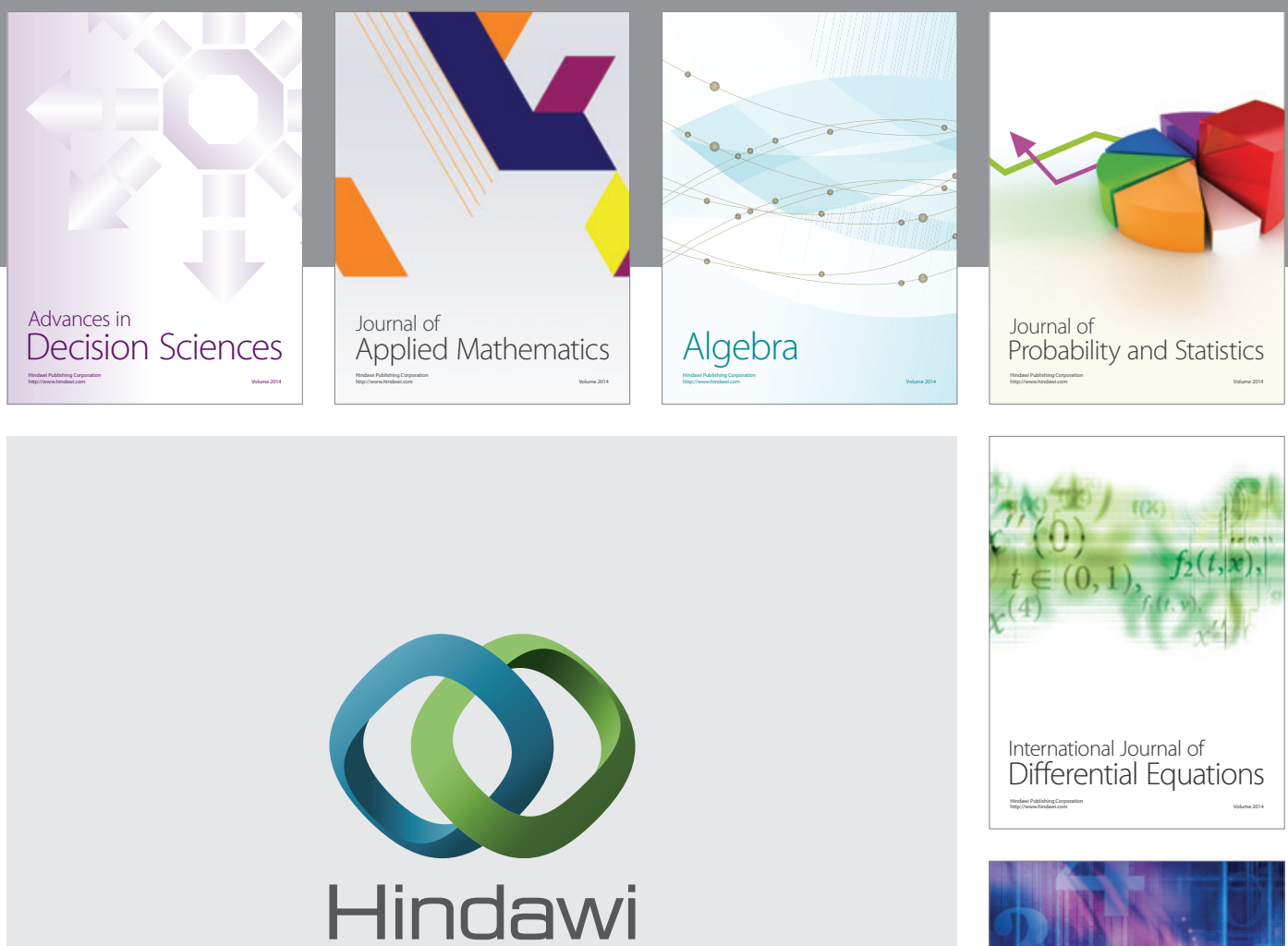

Submit your manuscripts at http://www.hindawi.com
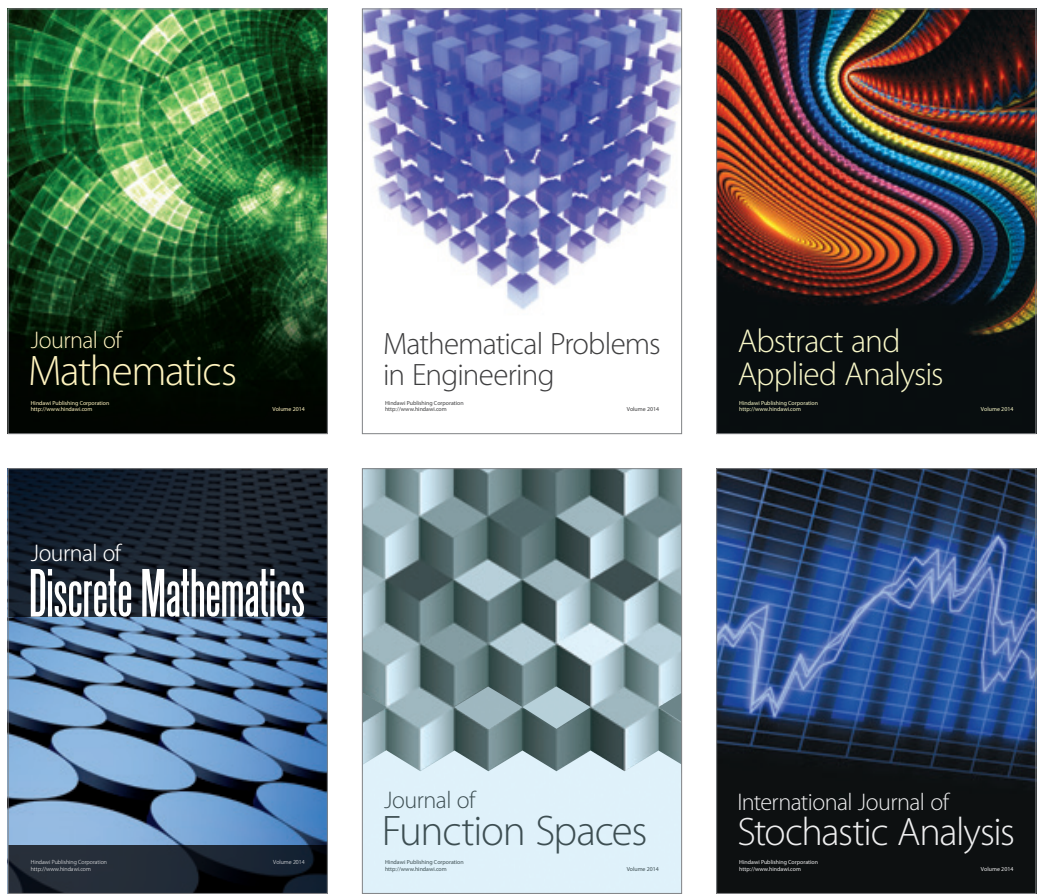

Journal of

Function Spaces

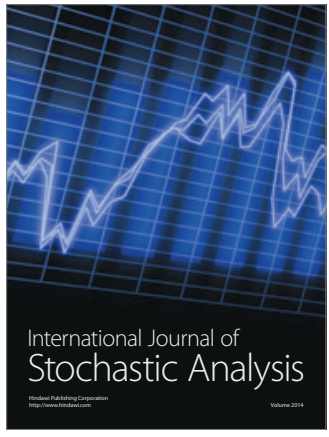

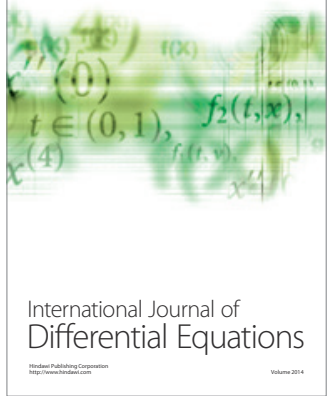
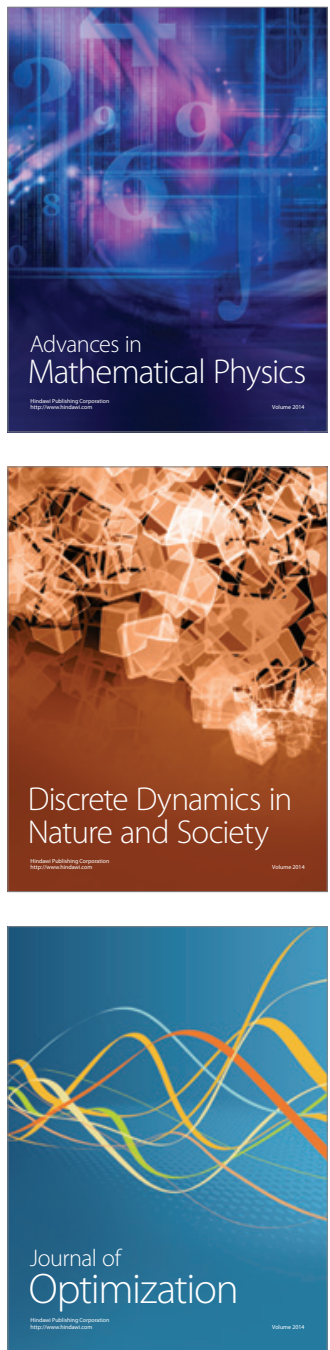